\title{
The role of the complement system in cancer
}

\author{
Vahid Afshar-Kharghan \\ Section of Benign Hematology, University of Texas, MD Anderson Cancer Center, Houston, Texas, USA.
}

\begin{abstract}
In addition to being a component of innate immunity and an ancient defense mechanism against invading pathogens, complement activation also participates in the adaptive immune response, inflammation, hemostasis, embryogenesis, and organ repair and development. Activation of the complement system via classical, lectin, or alternative pathways generates anaphylatoxins ( $\mathrm{C} 3 \mathrm{a}$ and $\mathrm{C} 5 \mathrm{a}$ ) and membrane attack complex (C5b-9) and opsonizes targeted cells. Complement activation end products and their receptors mediate cell-cell interactions that regulate several biological functions in the extravascular tissue. Signaling of anaphylatoxin receptors or assembly of membrane attack complex promotes cell dedifferentiation, proliferation, and migration in addition to reducing apoptosis. As a result, complement activation in the tumor microenvironment enhances tumor growth and increases metastasis. In this Review, I discuss immune and nonimmune functions of complement proteins and the tumor-promoting effect of complement activation.
\end{abstract}

\section{Introduction}

The complement system is a cascade of serine proteases encoded by genes originating from the same ancestral genes as coagulation proteins (1). Like the coagulation system, complement activation involves several steps, is tightly regulated, and requires both plasma and membrane proteins $(2,3)$. Many complement proteins possess dual functions that provide crosstalk between the complement system and other effector and regulatory systems. As a result, the complement system participates in adaptive immunity, hemostasis, neuroprotection and synaptic pruning, and organ development in addition to its role in innate immunity. It is also involved in a diverse array of pathologic conditions, such as thrombotic disorders, autoimmune disorders, schizophrenia, alloimmune responses including allograft rejection and graft-versushost disease, and cancer.

The complement system's role in fighting invasive pathogens has been extensively studied $(4,5)$, but recent discoveries provide new perspectives on the complement system's function in the extravascular and interstitial tissue compartment. These discoveries illustrate an important role for complement proteins in cellcell and stroma-cell communications. In this Review, I briefly discuss activation, regulation, immune, and nonimmune functions of the complement system to provide a framework for examining the role of complement in cancer.

\section{Activation of the complement system}

The complement system is activated by three major pathways: the classical pathway, via antigen-antibody complexes; the alternative pathway, via any permissive surfaces; and the lectin pathway, via binding of pattern-recognizing mannose-binding lectins (MBLs) to carbohydrate ligands on the surface of pathogens (Figure 1 and refs. 6-9). The convergence point for all complement activa-

Conflict of interest: The author has declared that no conflict of interest exists. Reference information: J Clin Invest. 2017;127(3):780-789.

https://doi.org/10.1172/JCI90962. tion pathways is the formation of the $\mathrm{C} 3$ convertase complex on the surface of targeted cells, summarized in Figure 1, A-C. After forming C3 convertase, complement is able to carry out its effector functions.

In all three complement activation pathways, C3 convertase complex cleaves C3 molecules to C3a, one of the two major anaphylatoxins, and to $\mathrm{C} 3 \mathrm{~b}$, a potent opsonin. Binding of $\mathrm{C} 3 \mathrm{~b}$ molecules to the surface of cells or cell debris in a process called opsonization marks them for phagocytosis by macrophages. Surface-bound $\mathrm{C} 3 \mathrm{~b}$ and its degradation products are ligands for complement receptors CR1, CR3, and CR2 that are expressed on myelomonocytic cells, lymphocytes, and follicular dendritic cells. Binding of $\mathrm{C} 3 \mathrm{~b}$ and its degradation products to correspondent receptors are crucial to cell-cell interactions in the innate and adaptive immune responses and in the removal of complement-coated apoptotic and necrotic cells.

Propagation of complement activation by C3 convertase results in the generation of the $\mathrm{C} 5$ convertase complex on the cell surface. C5 convertase then cleaves C5 to C5a and C5b. C5a is a potent anaphylatoxin and recruits neutrophils to areas of inflammation and tissue damage. C5b forms a complex with C6 and C7 that may insert into cell membrane, and subsequently be joined by C8 and multiple C9 to form the membrane attack complex (MAC or C5b-9 complex; Figure 1D). Deposition of an adequate number of MACs disrupts the phospholipid bilayer of the cell membrane, leading to massive calcium influx, loss of mitochondrial membrane potential, and cell lysis. However, MAC deposition at sublytic concentrations on cell membrane has a different result, activating intracellular signal transduction and cell proliferation (10). Eukaryotic cells have developed several defense mechanisms to counteract the dire consequences of MAC accumulation at the cell surface, including expression of complement regulatory proteins (CRPs) that disassemble MAC (i.e., CD59, vitronectin, and clusterin), and endocytosis or shedding of MAC from the cell surface.

Thus, the three main consequences of complement activation are tagging of cells by $\mathrm{C} 3 \mathrm{~b}$ degradation products for phagocytosis; chemotaxis of inflammatory cells in response to $\mathrm{C} 3 \mathrm{a}$ and $\mathrm{C} 5 \mathrm{a}$; and 


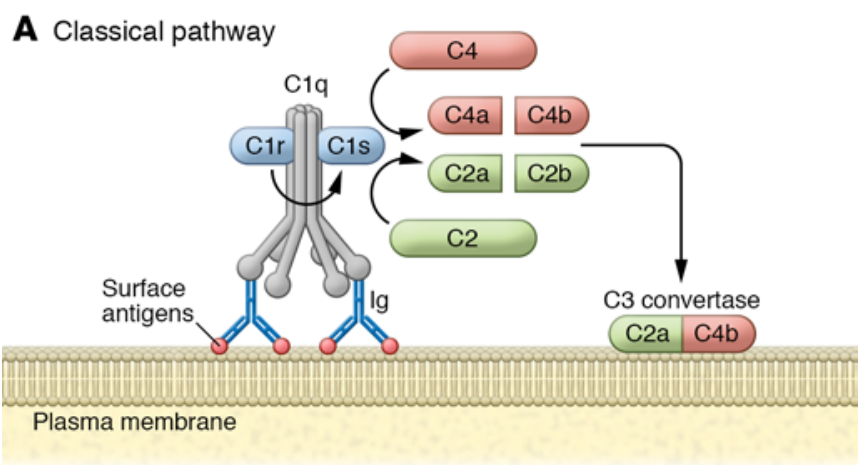

B Lectin pathway

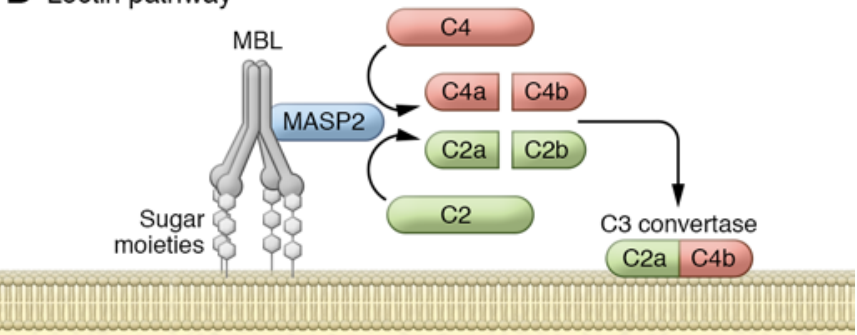

C Alternative pathway

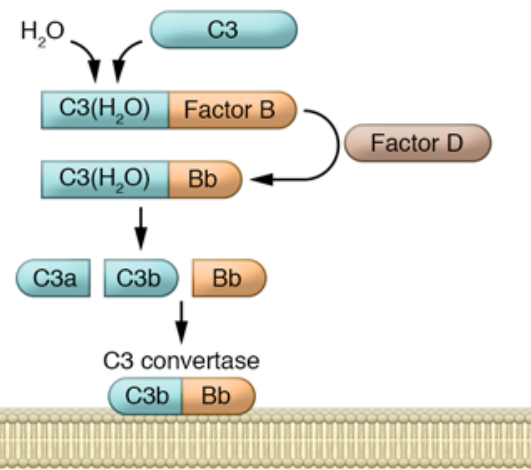

Generation of $\mathrm{C} 5$ convertase
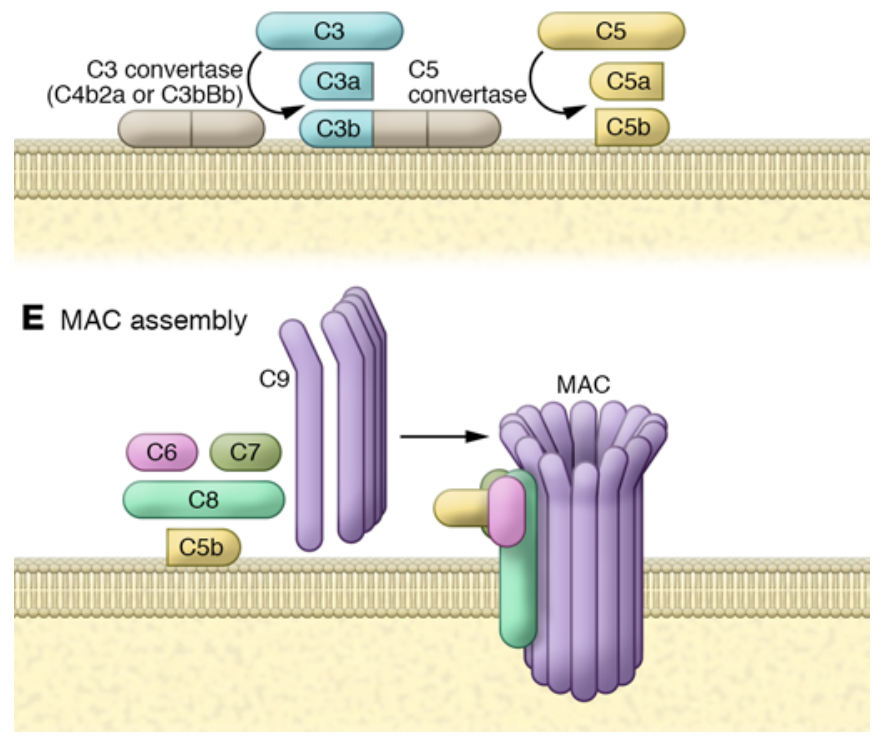

Figure 1. Complement activation. (A) The classical pathway is initiated by a complement-fixing antibody binding to an antigen on targeted cells. C1q binds to the antibody's Fc domain in the antibody-antigen complex. C1r and C1s assemble on C1q, C1r cleaves and activates C1s, and activated C1s cleaves $C 4$ and $C 2$ into $C 4 \mathrm{~b}$ and $\mathrm{C2}$ a, respectively. $\mathrm{C} 4 \mathrm{~b}$ and $\mathrm{C} 2 \mathrm{a}$ form the $\mathrm{C3}$ convertase $\mathrm{C} 4 \mathrm{bC2}$. (B) In the lectin pathway, MBL binds to repetitive sugar moieties such as mannose. MBL and MASP2 then form a C1-like complex. Activated MASP2 in MBL-MASP2 complex cleaves $C 4$ and $C 2$ and generates C3 convertase (C4bC2a). (C) In the alternative pathway, small amounts of hydrolyzed plasma $\mathrm{C3}[\mathrm{C} 3(\mathrm{H} 2 \mathrm{O})]$ bind to factor $\mathrm{B}$, which forms the $\mathrm{C3}(\mathrm{H} 2 \mathrm{O})$ $\mathrm{Bb}$ complex with help from factor D. C3(H2O)Bb cleaves additional plasma C3 to generate highly active $C 3 \mathrm{~b}$, which binds to cell the surface. On a complement-activating surface, $\mathrm{C} 3 \mathrm{~b}$ binds $\mathrm{Bb}$ (produced by factor $\mathrm{D}$-mediated cleavage of factor B) and generates C3bBb (the alternative pathway's C3 convertase). (D) Regardless of the initiation steps, C3 convertase deposits additional C3b molecules and generates C3a. If it remains intact, C3 convertase binds to additional $C 3 \mathrm{~b}$ to generate $C 5$ convertase. $C 5$ convertase cleaves $C 5$ to generate $\mathrm{C} 5 \mathrm{~b}$. (E) $\mathrm{C} 5 \mathrm{~b}$ binds to $\mathrm{C} 6, \mathrm{C} 7$, and $\mathrm{C} 8$, forming a $\mathrm{C} 5 \mathrm{~b}-8$ complex, which polymerizes several $C 9$ molecules, forming the cytolytic MAC.

MAC-mediated cell lysis. As described below, complement activation end products affect tumor growth by altering cancer cell behavior and modulating the immune response to the tumor.

\section{Regulation of the complement system}

The complement system's ability to cause cellular damage is strictly controlled at several steps, both in the fluid phase and on the cell surface (6). In the classical and lectin pathways, C1 inhibitor (C1INH) binds to and inactivates C1r, C1s, and MBLassociated serine proteases (MASPs). The activities of other CRPs can be categorized into two major groups: (a) decay-accelerating activity, which breaks up the $\mathrm{C} 3$ convertase complex, as can be seen in C4-binding protein (C4bp), CR1, decay-accelerating factor (DAF, also known as CD55), and factor $\mathrm{H}$; and (b) membrane cofactor activity, which acts as a cofactor for the factor I-mediated cleavage of $\mathrm{C} 3 \mathrm{~b}$ or $\mathrm{C} 4 \mathrm{~b}$ to their inactive degradation products, $\mathrm{iC} 3 \mathrm{~b}$ and $\mathrm{iC} 4 \mathrm{~b}$, respectively. CRPs with membrane cofactor activity include C4bp, CR1, membrane cofactor protein (MCP, or CD46), and factor $\mathrm{H}$.

Another important CRP is CD59, which is expressed on many different cell types and prevents assembly of MAC on the cell membrane.

The anaphylatoxins C3a and C5a are complement activation products that are rapidly inactivated in plasma by carboxypeptidases, particularly carboxypeptidase N (11). CRPs are overexpressed by many cancer cells and may be used as potential therapeutic targets.

\section{Immune function of the complement system}

The complement system is an ancient defense mechanism preceding adaptive immunity (12). It can be activated by pattern-recognition molecules and natural antibodies (13). Complement system activation and generation of anaphylatoxins orchestrate an inflammatory response to pathogens $(12,14)$. Anaphylatoxins activate macrophages, neutrophils, mast cells, basophils, and eosinophils, resulting in their degranulation and the production of cytokines, which in turn causes vasodilation, increases vascular permeability, and enhances neutrophil extravasation and chemotaxis (13). 
The complement system links innate immunity to adaptive immunity. Complement deficiency impairs both $\mathrm{B}$ and $\mathrm{T}$ cell responses (15). The effect of complement on the $\mathrm{B}$ cell response is mediated by CR2 on B cells and follicular dendritic cells. Activation of the classical pathway on the surface of an antigen tags that antigen with $\mathrm{C} 3 \mathrm{~d}$, enabling its binding to CR2 on B cells. CR2, CD19, and CD81 form a B cell coreceptor complex, and CR2 engagement with C3d enhances signaling through antigen-encountered $\mathrm{B}$ cell receptors and decreases the activation threshold of B cells $(12,15)$. The interaction between CR2 on follicular dendritic cells and C3d on antigens is important for antigen presentation to naive and primed $\mathrm{B}$ cells in the germinal center of lymph nodes, in the maturation of B cells, and in the generation of memory B cells.

The role of complement proteins in the cognate interaction between antigen-presenting cells (APCs) and T cells is important in the $\mathrm{T}$ cell immune response (16). In addition to systemic production in the liver, complement proteins are also produced locally by T cells and APCs (17-20). The effects of complement proteins on activation, proliferation, and differentiation of T cells are mediated by the local complement activation, by production of $\mathrm{C} 3 \mathrm{a}$ and $\mathrm{C} 5 \mathrm{a}$ at the interface of T cells and APCs, and through anaphylatoxin receptors on T cells and APCs (17-19, 21, 22). Reducing the number of $\mathrm{C} 3 \mathrm{a}$ and $\mathrm{C} 5 \mathrm{a}$ receptors (C3aR and $\mathrm{C} 5 \mathrm{aR}$, respectively) on T cells or APCs impairs T cell immunity.

Complement proteins and receptors are involved in different stages of the interaction between APCs and T cells. APCs produce C3 and express C3aR and C5aR, both of which are essential for their maturation and differentiation (19) and for effective antigen presentation to T cells $(17,23,24)$. C3- or C3aR-deficient APCs are much less potent in inducing a $\mathrm{T}$ cell immune response compared with WT APCs $(19,25)$. After APCs present antigen to $\mathrm{T}$ cells, $\mathrm{C} 5 \mathrm{aR}$ on the $\mathrm{T}$ cells is required for their proliferation. Binding of C5a to C5aR on $\mathrm{T}$ cells has both antiapoptotic and pro-proliferative effects (22).

\section{Nonimmune function of the complement system}

Cell-cell and stroma-cell interactions mediated by complement proteins regulate several physiologic processes, such as collective cell migration during embryogenesis (26), synaptic pruning during brain development (27-30), cell proliferation and differentiation during liver regeneration (31) and bone development $(32,33)$, and hematopoietic stem cell migration and engraftment during hematopoiesis (34).

\section{Complement and cancer}

The surge of interest in cancer immunotherapy is mainly focused on manipulating function or number of cytotoxic T cells. However, two important reasons justify studying the role of complement activation in cancer progression and the effect of complement manipulation in cancer therapy. First, the complement system is an important component of the inflammatory response, and inflammation is involved in various stages of tumorigenesis and cancer progression (35). Second, complement activation regulates adaptive immune response (15) and might have a role in regulating $\mathrm{T}$ cell response to tumors.
Complement system in inflammation and tumorigenesis. Tumor-promoting inflammation has an important role in carcinogenesis and cancer progression (36-38). A series of elegant experiments established that activation of the complement system is an important component of tumor-promoting inflammation. Bonavita et al. showed that C3-deficient mice were protected against chemical carcinogenesis in mesenchymal and epithelial tissues (39), mainly because of reduced inflammation. Authors identified a humoral component of innate immunity, the long pentraxin PTX3, as an important negative regulator of inflammation and complement activation. PTX3-deficient mice were susceptible to chemical carcinogenesis, displaying an increased number of tumor-associated macrophages with M2 phenotype and increased concentration of CCL2 chemokine inside tumors. The tumor-promoting inflammation induced by PTX3 deficiency was complement-dependent and completely reversed after removal of $\mathrm{C} 3$, as manifested by a reduction in the susceptibility of $\mathrm{Ptx}^{-/-} \mathrm{C3}^{-/-}$mice to chemical carcinogenesis. Similarly, treatment with the C5aR antagonist PMX-53 reversed the susceptible phenotype of Ptx $3^{-1-}$ mice without affecting the rate of tumorigenesis in $P t x 3^{+/+}$mice.

Activation and regulation of complement pathways in tumors. Expression of complement and CRPs is increased in malignant tumors and cancer cell lines (summarized in Table 1). Complement proteins, C3 degradation products, and complement activation products (i.e., C5a, C3a, and C5b-9) are easily detectable in various types of cancer, consistent with complement activation inside these tumors.

The main pathway involved in activation of complement inside tumors is unclear, and evidence supports activation of each complement pathway in malignant tumors (40). To make matters more complicated, cancer cell membrane-bound serine proteases can also cleave C5 and generate C5a without complement activation (41). Additionally, complement proteins expressed in tumors might also play a role in cancer progression independent of complement activation, as was shown for C1q in a syngeneic murine model of melanoma, where C1q expression affected angiogenesis, tumor progression, and metastasis (42). In this murine model, C1q was expressed in endothelial cells, spindle-shaped fibroblasts, and tumor-infiltrating myeloid cells independently of C4. Lack of C4 coexpression in $\mathrm{C} 1 \mathrm{q}$-expressing tumors hints at a role for $\mathrm{C} 1 \mathrm{q}$ in tumor progression independent of the classical pathway.

Expression of CRPs, including both membrane proteins (CD55, $\mathrm{CD} 59$, MCP, or CD46) and soluble proteins (factor $\mathrm{H}$ and factor $\mathrm{H}$-like proteins), is increased in cancer cells (43), although the overexpression of CRPs is heterogeneous among different cancer types and even between different tumor specimens of the same type of cancer (44).

One interpretation of the presence of both complement activation products and CRPs in tumors is that complement activation is a host defense mechanism against cancer, and cancer cells resist complement attack by overexpressing CRPs. However, as discussed later in this Review, several recent studies do not support this interpretation and suggest another scenario in which local complement activation inside tumors enhances tumor growth.

Complement activation: antitumor or protumor? Evidence for the effects of complement on malignant transformation of epithelial cells and progression of cancer has evolved based on several recent studies showing complex and sometimes contradictory findings. 
Table 1. Complement proteins in cancer

Complement proteins C3
Malignancy types/models

Cervical cancer (murine models)

Ovarian cancer (murine models)

Ovarian cancer (murine models)

Cervical intraepithelial neoplasia III (patient samples)

Non-small-cell lung cancer (patient samples)

Clioblastoma multiforme (patient samples)

Melanoma

[3a/C3aR

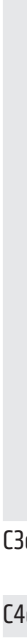

C4d

C5b-9

C1q

$[5 a / C 5 a R$

$3 d$

Biliary and colon cancer (cell lines)

Biliary and colon cancer (cell lines)

Cervical cancer (murine models)

Ovarian cancer (murine models)

Ovarian cancer (murine model)

Melanoma

Colon cancer (patient samples)

Oral and oropharyngeal squamous cell cancer (patient samples)

Gastric adenocarcinoma (patient samples)

Ovarian cancer (patient samples)

Breast cancer

Thyroid cancer

Ovarian cancer (murine models)

Melanoma (murine models and cell line), breast, colon, lung, and pancreatic cancer (cell lines)

Clioblastoma multiforme (patient samples)

Cervical cancer (murine models)

MBL-MASP

Colorectal cancer (patient samples)

Colorectal cancer (patient samples)

Clioblastoma multiforme (patient samples)

Factor $\mathrm{H}$
Effect of complement proteins

Refs.

Activation of classical complement pathway inside the tumor

Promote tumor growth

Immunomodulatory effect by increasing MDSCs and reducing $\mathrm{CD}^{+}$inside the tumor

Complement activation inside the tumor

Promote tumor growth

Autocrine effect mediated by anaphylatoxins generated in tumors

and their receptors on cancer cells

Promote tumorigenesis and tumor growth

Promote angiogenesis

Present in patients' sera

A biomarker for tumor progression

Tissue deposition

Correlates with prognosis

Tumor tissue deposition

Autocrine inhibition of $\mathrm{CD} 8^{+}$TILs

C3aR deficiency or C3aR antagonist reduces tumor growth

Promote tumor growth

Increase in C3a serum concentration

Increase [3a concentration in ascites

Promote EMT

Reduce IL-10 synthesis by CD8 ${ }^{+}$TILs

C5a generated by cancer cells

Promote tumor growth

Immunomodulatory effect

Blockade of C5aR significantly reduced MDSCs and immunomodulators

such as ARG1, CTLA-4, LL-6, IL-10, LAG3, and PDL1 (B7H1)

Increase cell motility and invasiveness

C5a is generated by a serine protease on the surface of cancer cells independent of complement activation

Promote tumor growth

Immunomodulatory effect by increasing $C D 8^{+} T$ cells and reducing MDSCs inside the tumor

Promote tumor growth

Autocrine effect through C5aRs on cancer cells

Promote tumorigenesis and tumor growth

Promote angiogenesis

Reduce IL-10 synthesis by CD8 ${ }^{+}$TILs

systemic venous blood

Tumor tissue deposition

Tumor tissue deposition correlating to the clinical stage 100

Increase in concentration of soluble C5b-9 in ascites

Tumor tissue deposition

Tumor tissue deposition

102103

Tumor tissue deposition

73

Tumor tissue deposition (independent of C4 deposition, mainly on cells

in the tumor microenvironment with mesenchymal origin)

Promote angiogenesis, progression of the tumor, and metastasis

(independent of complement activation)

Tumor tissue deposition

Tumor tissue deposition

MBL concentration and MBL-MASP activity increased in patients' sera 105

High MASP2 level in serum predicts recurrence and short survival 106

MBL deficiency (polymorphic variant) associated with a lower risk of 92 glioblastoma multiforme

Tumor tissue expression

A biomarker for tumor progression

Increase in urine concentration in patients

(1)


This complexity is similar to the complex role of inflammation in cancer (45). Although inflammatory cells and cytokines are important in immune surveillance, exemplified by the benefit of bacillus Calmette-Guérin therapy in early stages of bladder cancer, chronic inflammation promotes carcinogenesis and tumor growth. Even immune cells, such as macrophages, can have both pro- and antitumor phenotypes. Despite this multifaceted picture, most evidence points toward a protumor effect of chronic inflammation (45).

The long-held view of complement activation as an antitumor defense mechanism is based on two main concepts: first, the complement system's participation in immune surveillance against malignant cells, and second, complement-dependent cytotoxicity of therapeutic monoclonal antibodies. I will discuss these concepts below, and summarize new information pointing toward a protumor effect of complement activation inside tumors.

Complement and immune surveillance. The complement system's ability to distinguish self from non-self makes it an important part of the innate immune response to invading pathogens (46). Expression of non-self antigens and lack of CRPs on microbes make them optimal targets for complement detection and, later on, complement-mediated elimination. Similarly, expression of danger signals and neoantigens by apoptotic cells and cellular debris optimizes their detection and removal by the complement system. Cancer cells, on the other hand, mostly express the same proteins as their normal epithelial cell counterparts, albeit occasionally with a different density. Furthermore, overexpression of CRPs by cancer cells limits immune surveillance by the complement system $(3,43,46,47)$. Putting these findings together, one can conclude that cell-mediated immunity plays a more important role than humoral immunity in immune surveillance against cancer cells $(48,49)$, and effectiveness of complement in early detection and elimination of cancer cells is uncertain (50).

Complement-dependent cytotoxicity. Complement activation was considered detrimental to cancer cells via complementdependent cytotoxicity, which causes cancer cell lysis via MAC accumulation or phagocytosis of opsonized cancer cells by macrophages and neutrophils. Complement-dependent cytotoxicity is considered to be the main mechanism for the effectiveness of antitumor monoclonal antibodies. Rituximab, an anti-CD20 antibody against malignant $\mathrm{B}$ cells, is among the oldest and most widely used therapeutic monoclonal antibodies. Although in vitro and in vivo studies show that rituximab activates the classical complement pathway $(51,52)$, the notion that its therapeutic benefits are mainly mediated by induction of complement attack on malignant B cells is questionable. In fact, the antitumor effect of rituximab was inhibited by deposited complement proteins on $\mathrm{B}$ cells (53), and was enhanced in complement-deficient mice (54). Therefore, the extent to which complement-dependent cytotoxicity contributes to other immunologic effects of rituximab, i.e., antibody-dependent cellular cytotoxicity and antibody-dependent phagocytosis, is unknown. Other studies on the therapeutic mechanism of rituximab also showed a complement-independent, proapoptotic effect mediated by cross-linking of CD2O (55), as well as antiproliferative and antisurvival effects that were mediated by inhibition of B cell receptors (56). Furthermore, many in vitro antitumor effects of complement-fixing antibodies on cancer cell lines were not reproduced in vivo (57).
Complement activation promotes tumor growth. Considering that complement is not efficient in immune surveillance against cancer cells and that the main antitumor effect of monoclonal antibodies might not arise from complement activation, the data supporting an antitumor role for complement activation are scant. The question remains: If complement does not attack cancer cells, how does local complement activation and deposition of complement proteins affect tumors? To understand the consequence of complement activation inside tumors, it is helpful to reexamine the biological functions of complement activation products. C3b and its degradation products binding to CR1, CR2, and CR3 provide ligands and receptors for cell-cell and stroma-cell interactions in many physiologic and pathologic conditions. Complement activation generates $\mathrm{C} 3 \mathrm{a}$ and $\mathrm{C} 5 \mathrm{a}$ and MAC. The anaphylatoxin receptors $\mathrm{C} 3 \mathrm{aR}$ and $\mathrm{C} 5 \mathrm{aR}$ are $\mathrm{G}$ protein-coupled receptors present on many cell types, including lymphocytes, monocytes/macrophages, myeloid cells, hematopoietic stem cells, mesenchymal cells, and epithelial cells, including cancer cells. Anaphylatoxin receptor signaling has been studied extensively (58). Activation of C5aR promotes a range of responses depending on the cell type. Relevant to its role in cancer, $\mathrm{C} 5 \mathrm{aR}$ activation generates prosurvival and antiapoptotic responses. For example, C5a binding to C5aR decreases apoptosis in neutrophils (59) and T cells (22), and increases cell proliferation in endothelial (60) and colon cancer cell lines (61). Activation of C3aR plays an important role in guiding collective cell migration (26) and epithelial-mesenchymal transition $(62,63)$, both important mechanisms in metastasis. In a sublytic density, MAC accumulation on the cell membrane promotes cell proliferation (64) and differentiation, inhibits apoptosis $(10,65)$, and protects cells against complement-mediated lysis (66).

Markiewski et al. showed that the activation of the classical complement pathway inside implanted orthotopic tumors in mice enhanced tumor growth (67). Complement's progrowth effect on tumors was C5a-dependent and was eliminated in C5aR-deficient mice and in WT mice treated with a C5aR antagonist. C5a modulates the immune response to tumors by acting as a chemotactic factor, increasing infiltration of myeloid-derived suppressor cells (MDSCs) and reducing the number of $\mathrm{CD} 8^{+}$cytotoxic T cells inside tumors. MDSCs are immature myeloid cells that increase in blood, bone marrow, and spleen of tumor-bearing mice and cancer patients $(68,69)$ and assist tumor cells in evading the antitumor immune response. MDSCs reduce proliferation and increase apoptosis in $\mathrm{CD}^{+} \mathrm{T}$ cells by generating ROS and reactive nitrogen species (70). Depletion of CD8 ${ }^{+} \mathrm{T}$ cells in mice eliminated the protective effect of complement deficiency against tumor growth. In summary, this study showed that the immunomodulatory effect of activated classical complement pathway inside tumors enhances tumor growth. The origin of complement proteins was the host, but activation of complement occurred inside the tumor microenvironment, and the final effect on the tumor was an indirect immunomodulatory effect mediated by MDSCs (Figure 2).

In a follow-up study, Nunez-Cruz et al. investigated complement's role in tumorigenesis in a murine model of spontaneous ovarian cancer $(71,72)$. C3 or C5aR deficiency in these mice prevented the development of ovarian tumors, permitting no tumors or only small and poorly vascularized tumor formation (71). C3 deficiency was associated with a change in the immune profile of leukocytes 


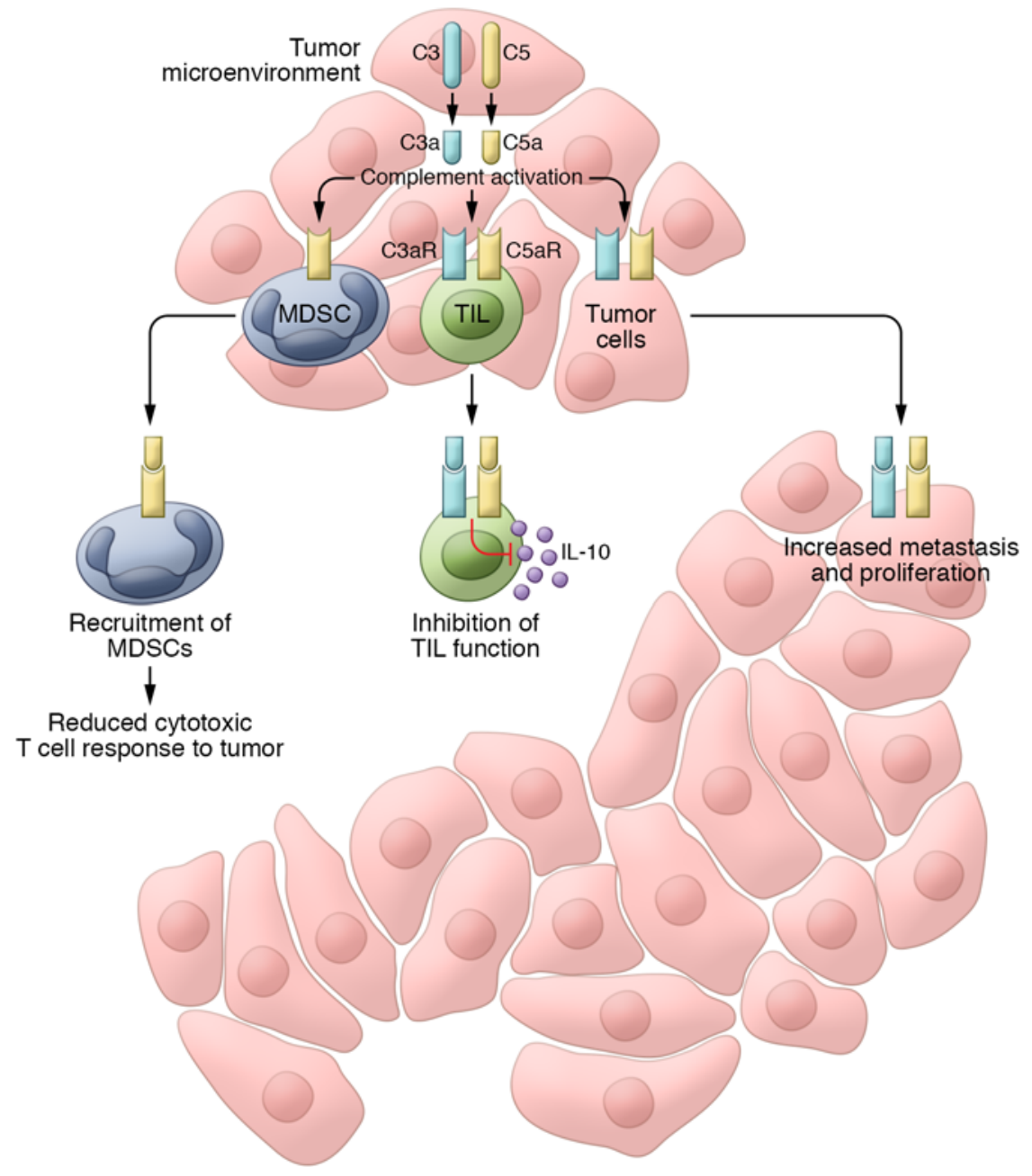

Figure 2. Effect of complement activation in the tumor microenvironment. Activation of the complement system inside tumors releases $\mathrm{C} 5 \mathrm{a}$ and $\mathrm{C} 3 \mathrm{a}$ into the tumor microenvironment and promotes tumor growth. $\mathrm{C5}$ a attracts myeloid cell, including MDSCs, into the tumor. MDSCs then reduce cytotoxic $T$ cell responses to the tumor by inducing apoptosis and inhibiting CD8 ${ }^{+}$ TILs via generation of ROS and reactive nitrogen species and depletion of arginine. In melanoma, secretion of $\mathrm{C} 3$ by CD8 ${ }^{+}$TILs and complement activation in the vicinity of these cells reduce IL-10 production by TILs and inhibit their function. Some cancer cell types secrete complement proteins into the tumor microenvironment and initiate an autocrine loop that increases cell proliferation and promotes metastasis. The effect of complement activation on MDSCs, TILs, and cancer cells is mediated by the $\mathrm{C} 5 \mathrm{a}$ and $\mathrm{C} 3 \mathrm{a}$ receptors ( $C 5 a R$ and $C 3 a R)$ on these cells.

infiltrating into the tumors, but C5aR deficiency reduced ovarian tumor size without altering the immune profile of infiltrating leukocytes. This result suggested the existence of a protumor effect of complement that was independent of its immunomodulatory effect.

We investigated the effect of complement in murine models of ovarian cancer and confirmed activation of complement in the tumor microenvironment (73). However, complement proteins detected inside ovarian tumors originated not from the host, but from tumor cells themselves. Complement activation products were present even inside tumors implanted in C3-deficient mice lacking a functional complement system. Although orthotopic ovarian cancer tumors in C3-deficient mice reached to the same size as those in WT mice, reducing $\mathrm{C} 3$ or $\mathrm{C} 5$ production in cancer cells significantly reduced the tumor growth independent of the host's complement sufficiency status. C3 synthesis can be detected in malignant epithelial cells originating from several different organs, particularly lung and ovary. Inhibiting synthesis of complement proteins in cancer cells altered the immune profile of leukocytes infiltrating into tumors, manifested by an increase in the number of $\mathrm{CD}^{+} \mathrm{T}$ cells and reduction in myeloid cells. However, immunomodulatory effect of complement inhibition was not the main mechanism responsible for the observed reduction in tumor growth. Inhibiting complement protein synthesis in cancer cells implanted in $\mathrm{CD} 8^{+} \mathrm{T}$ cell-deficient mice reduced tumor growth to the same magnitude as in WT mice. We investigated the possibility of an autocrine stimulation of cancer cells as a result of complement activation. Anaphylatoxin receptors are present on ovarian cancer cells, and stimulation of these receptors by C3a or C5a agonist peptides increased proliferation and invasiveness of ovarian cancer cells in vitro. Furthermore, knockdown of these receptors on cancer cells reduced growth of orthotopic ovarian tumors in mice. Our studies showed that local complement activation inside the tumor microenvironment enhances tumor growth via a direct autocrine effect on ovarian cancer cells increasing cell proliferation (Figure 2).

In a murine model, Wang et al. reported another mechanism for the progrowth effect of complement activation in melanoma, showing that production of IL-10 by CD8 ${ }^{+}$tumor-infiltrating lymphocytes (TILs) is constitutively inhibited in an autocrine fashion by $\mathrm{C} 3$ originating from $\mathrm{CD} 8^{+}$TILs themselves, acting through C5aR and C3aR on the surface of these lymphocytes (74). C3aR and C5aR antagonists increased IL-10 production and activated CD8 ${ }^{+}$TILs that in turn reduce tumor growth. The IL-10-dependent antitumor activity of complement inhibitors in melanoma was independent of the PD-1/PD-L1 axis or MDSCs. This study provides evidence that local complement activation in the tumor microenvironment results in suppression of the immune response to melanoma by inhibiting $\mathrm{CD}^{+} \mathrm{TIL}$ function (Figure 2). 
The studies above describe different mechanisms by which complement activation in the tumor microenvironment can enhance tumor growth: (a) by altering the immune profile of tumor-infiltrating leukocytes, (b) by increasing cancer cell proliferation, and (c) by directly suppressing $\mathrm{CD} 8^{+}$TIL function. It is possible that different cancer types use different mechanisms to take advantage of ectopic complement activation inside tumors. For example, ovarian cancer cells synthesize a significant amount of complement proteins and initiate an autocrine loop resulting in increased cell proliferation by a direct effect of anaphylatoxins on cancer cells. Conversely, melanoma cells do not secrete complement proteins, and complement proteins produced by CD8 ${ }^{+}$TILs reduce their IL-10 production and antitumor activity.

An important question remains whether complement activation has any role in malignant transformation of normal cells or only affects the expansion of already established malignant clones. Most available data are based on orthotopic murine models of ovarian cancer or mice genetically engineered to develop ovarian cancer by overexpression of oncogenes. These studies showed that complement promotes growth and expansion of malignant tumors. Bonavita et al. showed that complement promotes malignant transformation of cells exposed to chronic inflammation induced by chemical carcinogens (39). However, additional studies are required to dissect the effect of early versus late stages of complement activation on various stages of oncogenesis.

\section{Overexpression of CRPs on cancer cells}

If complement activation promotes tumor growth and oncogenesis, why are CRPs overexpressed on cancer cells? One would expect that cancer cells, under selective pressure, downregulate expression of CRPs to benefit from complement activation. To reconcile these seemingly counterintuitive observations, we put forward the following hypothesis: Anaphylatoxins and sublytic concentrations of MAC promote tumor growth, but higher concentrations of MAC have a tumoricidal effect. As a result, cancer cells benefit from early stages of complement activation and production of anaphylatoxins, but actively inhibit generation of MAC. Cancer cells reduce MAC concentration by overexpressing CD59, the most consistently overexpressed CRP on different cancer cells (75) and the most effective membrane regulatory protein against complement-mediated lysis $(43,76,77)$, eliminating MAC from the cell surface through membrane vesiculation. Thus, from a therapeutic point of view, interventions that reduce complement activation or promote the generation of MAC inside tumors can be considered as logical options to counter the progrowth effect of complement on cancer.

\section{Complement activation in \\ epithelial-mesenchymal transition}

Epithelial-mesenchymal transition (EMT) occurs in physiologic processes such as embryogenesis and organ development, and in pathologic conditions including tissue fibrosis and metastasis $(78,79)$. Complement participates in EMT in murine models of renal injury and fibrosis $(62,80,81)$. We showed that complement activation inside tumors not only increases tumor growth but also enhances metastasis by promoting EMT in cancer cells. In ovarian cancer cells, the transcription factor TWIST1 upregulates C3 gene expression, generating $\mathrm{C} 3 \mathrm{a}$ in the tumor microenvironment, which binds to C3aR on ovarian cancer cells. We further showed that C3aR signaling increases EMT and decreases E-cadherin expression in ovarian cancer cells via a Krüppel-like factor 5dependent mechanism and promotes EMT and metastasis (63).

In addition to promoting metastasis, EMT also induces resistance to complement-dependent cytotoxicity in lung cancer cells by increasing expression of CD59 (82). Inhibition or knockdown of CD59 restored sensitivity of cancer cells to complementdependent lysis without altering the morphologic features or protein markers of EMT in these cells.

\section{Therapeutic potential of targeting complement activation in cancer}

Our understanding of the role of the complement system in cancer biology is evolving, changing our approach to the therapeutic use of reagents modifying the complement system. Traditional methods targeting cancer cells using antitumor antibodies to promote lysis of cancer cells by MACs require identification of tumor-specific antigens that either are expressed with a higher density on cancer cells than normal epithelial cells, or are only expressed on cancer cells. Antibodies against EGFR and CD2O are among the most successful therapeutic antibodies. Development of therapeutic antibodies was initially complicated by induction of an immune response to polyclonal antibodies developed in nonhuman hosts. Development of monoclonal murine antibodies; later, chimeric human-mouse antibodies; and recently, humanized antibodies (83) helps overcome this problem. However, a more important problem in harvesting complement-dependent cytotoxicity induced by therapeutic antibodies is overexpression of membrane CRPs by cancer cells that let cancer cells evade MAC-mediated cytolysis (43). As a result, blockade of membrane CRPs on cancer cells, alone or in conjunction with use of therapeutic antibodies, has been tried as another potential therapeutic strategy. Blocking CRPs reduces cancer cell proliferation in vitro (43) and tumor growth in mice (76). CD59 blocking antibodies or CD59 siRNA enhanced complement-mediated cytolysis induced by anti-EGFR monoclonal antibodies (trastuzumab and cetuximab) in human lung cancer cell lines (84). rILYd4, a recombinant protein inhibitor of CD59, increased sensitivity of malignant B cells to rituximab in vitro and in orthotopic murine models (85).

Membrane CRPs are universally expressed, and an important theoretical complication of blocking CRPs is exposing normal cells to complement-dependent cytotoxicity. For example, CD59 and CD55 protect red blood cells against complement-induced hemolysis, and blocking CD59 might cause hemolysis. Interestingly, administration of rILYd4 in mice was not associated with significant increases in hemolysis (85).

More recent studies showed a protumor effect of complement, and inhibition of complement activation in vitro or in murine models of cancer was investigated as a novel way to treat cancer. Understanding of the autocrine and paracrine effects of complement production and activation inside tumors versus its systemic immunomodulatory effect is not complete, but blocking complement activation or inhibiting C5aR and C3aR signaling inside tumors seems a reasonable approach. However, several questions and concerns regarding the therapeutic use of anticomplement reagents have not been addressed and require additional studies: 
Does systemic complement inhibition affect local production and activation of complement in the tumor microenvironment? Pharmacokinetic studies based on measurements of tissue concentration of various anticomplement reagents may resolve this issue, although the leakiness of tumor vasculature likely provides adequate tissue penetrance of these reagents.

Does complement have a protumor effect in many or only in a few types of cancer? Recent studies showed that complement activation enhances growth of ovarian, cervical, and non-small-cell lung cancer and sarcoma, and deposition of complement proteins is detectable in more cancer cell types (Table 1).

Which complement pathways are activated in cancer? Evidence supporting activation of classical, lectin, and alternative pathways in cancer exists. It is possible that in different types of cancer different pathways are functional. Therefore, it is more reasonable to target common complement proteins or receptors in antitumor therapies.

What are the effects of early (C3a and C5a) versus late (MAC) complement activation end products on cancer cells? If higher concentrations of C3a or C5a promote, and denser MAC deposit reduces, tumor growth, developing bispecific inhibitory antibodies targeting C5aR (or C3aR) and CD59 simultaneously may increase the potency of the antitumor effect of complement therapy.

Complement-dependent cytotoxicity has been considered an important component of the therapeutic benefit of monoclonal antibodies in malignant $B$ cell disorders; however, the effects of complement activation on white blood cell dyscrasia have not been studied. A few reports point to a prognostic significance of expression of complement genes in leukemic blasts $(86,87)$. More comprehensive studies on the role of complement activation in leukemia and lymphoproliferative disorders might reveal possible therapeutic benefits of anticomplement reagents in these disorders.

Eculizumab, a humanized anti-C5 monoclonal antibody, is currently available on the market and is used to treat paroxysmal nocturnal hemoglobinuria and atypical hemolytic uremic syndrome. A single i.v. infusion of eculizumab blocks complement activation in plasma for 2-3 weeks $(88,89)$, but its potency and half-life in the interstitial tissue are unknown. Eculizumab blocks generation of C5a and MAC, but would not affect synthesis and secretion of complement proteins by cancer cells or $\mathrm{C} 3 \mathrm{a}$ generation in the tumor microenvironment. Currently, no ongoing clinical trials are evaluating eculizumab in cancer patients; however, because of the clinical use of this reagent for other indications, we have a relatively clear picture of its side effect profile. Patients on eculizumab are at risk for developing infections with encapsulated microorganisms and should receive meningococcal vaccination before initiation of therapy. Lack of bone marrow suppression with eculizumab is a therapeutic advantage that can be used in designing clinical trials combining this reagent with chemotherapeutic reagents in cancer patients.

In a few animal studies, C5aR antagonists, including PMX53 , have been shown to be effective in reducing tumor size in mice $(67,71)$, but this or similar reagents have not entered into clinical practice yet. Targeting C5aR rather than C5 or C3 might have the potential benefit of leaving opsonization and MAC generation intact. Intact opsonization of bacteria would reduce the risk of infectious complications in individuals undergoing treatment, and the generation of lytic concentrations of MAC might have a tumoricidal effect. On the other hand, targeting C5aR has the disadvantage of leaving other complement effector molecules, such as C3a, uninhibited.

A potential advantage of using anticomplement reagents in cancer treatment is that they can be combined with traditional chemotherapies without increasing myelosuppression associated with chemotherapies; and combined with immune checkpoint inhibitors, because they have different targets. While checkpoint inhibitors increase proliferation of cytotoxic T cells, complement inhibitors decrease MDSCs infiltrating into the tumor microenvironment, reduce MDSC-induced T cell suppression, and enhance $\mathrm{T}$ cell function. Based on experiences collected with the clinical use of eculizumab, another advantage of complement inhibitors is their relatively few side effects.

Any therapeutic use of anticomplement therapies in solid or liquid tumors should be carefully balanced with possible interference of complement inhibition with the efficacy of other antitumor reagents: (a) The outcome of combining anticomplement reagents with monoclonal antibodies (such as cetuximab, rituximab, or trastuzumab) may depend on the importance of complement-dependent cytotoxicity in the function of these antibodies. (b) Chimeric antigen receptor (CAR) $\mathrm{T}$ cell therapies depend on in vitro expansion and in vivo proliferation of $\mathrm{T}$ cells. Complement inhibition may decrease the proliferation of CAR T cells in vivo and may reduce their efficacy.

\section{Conclusions}

By mediating cell-cell and cell-stroma interactions, complement proteins have several immune and nonimmune functions in both plasma and the extravascular interstitial tissue. Activation of the complement system in the tumor microenvironment enhances tumor growth via different mechanisms. Anticomplement reagents might have a place in the therapeutic armamentarium against cancer and, because of their limited non-myelosuppressive side effects and nonoverlapping pharmacodynamics, could be combined with traditional chemotherapies or immunotherapies.

\section{Acknowledgments}

This work is supported in part by NIH grant CA177909 (to VAK). The author thanks Michael Kroll for his valuable comments.

Address correspondence to: Vahid Afshar-Kharghan, Section of Benign Hematology, 2121 W. Holcombe Boulevard, Suite 7.20E, University of Texas, MD Anderson Cancer Center, Houston, Texas 77030, USA. Phone: 713.563.5267; E-mail:Vakharghan@mdanderson.org.
1. Krem MM, Di Cera E. Evolution of enzyme cascades from embryonic development to blood coagulation. Trends Biochem Sci. 2002;27(2):67-74.

2. Sjöberg AP, Trouw LA, Blom AM. Complement activation and inhibition: a delicate balance. Trends Immunol. 2009;30(2):83-90.
3. Zipfel PF, Skerka C. Complement regulators and inhibitory proteins. Nat Rev Immunol. 2009;9(10):729-740.

4. Walport MJ. Complement. First of two parts. NEngl JMed. 2001;344(14):1058-1066.

5. Walport MJ. Complement. Second of two parts.
NEngl J Med. 2001;344(15):1140-1144.

6. Holers VM. Complement and its receptors: new insights into human disease. Annu Rev Immunol. 2014;32:433-459.

7. Turner MW. The role of mannose-binding lectin in health and disease. Mol Immunol. 
2003;40(7):423-429.

8. Jack DL, Klein NJ, Turner MW. Mannose-binding lectin: targeting the microbial world for complement attack and opsonophagocytosis. Immunol Rev. 2001;180:86-99.

9. Frank MM. Complement in the pathophysiology of human disease. $N$ Engl J Med. 1987;316(24):1525-1530.

10. Tegla CA, et al. Membrane attack by complement: the assembly and biology of terminal complement complexes. Immunol Res. 2011;51(1):45-60.

11. Matthews KW, Mueller-Ortiz SL, Wetsel RA. Carboxypeptidase $\mathrm{N}$ : a pleiotropic regulator of inflammation. Mol Immunol. 2004;40(11):785-793.

12. Dunkelberger JR, Song WC. Complement and its role in innate and adaptive immune responses. Cell Res. 2010;20(1):34-50.

13. Markiewski MM, Lambris JD. The role of complement in inflammatory diseases from behind the scenes into the spotlight. Am J Pathol. 2007;171(3):715-727.

14. Ricklin D, Lambris JD. Complement in immune and inflammatory disorders: pathophysiological mechanisms. JImmunol. 2013;190(8):3831-3838.

15. Carroll MC, Isenman DE. Regulation of humoral immunity by complement. Immunity. 2012;37(2):199-207.

16. Kemper C, Atkinson JP. T-cell regulation: with complements from innate immunity. Nat Rev Immunol. 2007;7(1):9-18.

17. Strainic MG, et al. Locally produced complement fragments $\mathrm{C} 5 \mathrm{a}$ and $\mathrm{C} 3$ a provide both costimulatory and survival signals to naive $\mathrm{CD} 4^{+} \mathrm{T}$ cells. Immunity. 2008;28(3):425-435.

18. Raedler H, Yang M, Lalli PN, Medof ME, Heeger PS. Primed CD8(+) T-cell responses to allogeneic endothelial cells are controlled by local complement activation. Am J Transplant. 2009;9(8):1784-1795.

19. Peng $Q$, et al. Local production and activation of complement up-regulates the allostimulatory function of dendritic cells through C3a-C3aR interaction. Blood. 2008;111(4):2452-2461.

20. Pratt JR, Basheer SA, Sacks SH. Local synthesis of complement component $\mathrm{C} 3$ regulates acute renal transplant rejection. Nat Med. 2002;8(6):582-587.

21. Sacks SH. Complement fragments C3a and C5a: the salt and pepper of the immune response. Eur JImmunol. 2010;40(3):668-670.

22. Lalli PN, Strainic MG, Yang M, Lin F, Medof ME, Heeger PS. Locally produced C5a binds to $\mathrm{T}$ cell-expressed C5aR to enhance effector T-cell expansion by limiting antigen-induced apoptosis. Blood. 2008;112(5):1759-1766.

23. Jacquier-Sarlin MR, Gabert FM, Villiers MB, Colomb MG. Modulation of antigen processing and presentation by covalently linked complement $\mathrm{C} 3 \mathrm{~b}$ fragment. Immunology. 1995;84(1):164-170.

24. Kerekes K, Cooper PD, Prechl J, Józsi M, Bajtay Z, Erdei A. Adjuvant effect of gamma-inulin is mediated by $\mathrm{C} 3$ fragments deposited on antigen-presenting cells. J Leukoc Biol. 2001;69(1):69-74.

25. Zhou W, Medof ME, Heeger PS, Sacks S. Graft-derived complement as a mediator of transplant injury. Curr Opin Immunol. 2007;19(5):569-576.
26. Carmona-Fontaine C, et al. Complement fragment C3a controls mutual cell attraction during collective cell migration. Dev Cell. 2011;21(6):1026-1037.

27. Stevens B, et al. The classical complement cascade mediates CNS synapse elimination. Cell. 2007;131(6):1164-1178.

28. Schafer DP, et al. Microglia sculpt postnatal neural circuits in an activity and complement-dependent manner. Neuron. 2012;74(4):691-705.

29. Bialas AR, Stevens B. TGF- $\beta$ signaling regulates neuronal C1q expression and developmental synaptic refinement. Nat Neurosci. 2013;16(12):1773-1782.

30. Sekar A, et al. Schizophrenia risk from complex variation of complement component 4 . Nature. 2016;530(7589):177-183.

31. Strey CW, et al. The proinflammatory mediators $\mathrm{C} 3 \mathrm{a}$ and $\mathrm{C} 5 \mathrm{a}$ are essential for liver regeneration. JExp Med. 2003;198(6):913-923.

32. Mastellos D, Lambris JD. Complement: more than a 'guard' against invading pathogens? Trends Immunol. 2002;23(10):485-491.

33. Del Rio-Tsonis K, Tsonis PA, Zarkadis IK, Tsagas AG, Lambris JD. Expression of the third component of complement, $\mathrm{C} 3$, in regenerating limb blastema cells of urodeles. JImmunol. 1998;161(12):6819-6824.

34. Reca R, et al. Functional receptor for C3a anaphylatoxin is expressed by normal hematopoietic stem/progenitor cells, and C3a enhances their homing-related responses to SDF-1. Blood. 2003;101(10):3784-3793.

35. Coussens LM, Werb Z. Inflammation and cancer. Nature. 2002;420(6917):860-867.

36. Coussens LM, Zitvogel L, Palucka AK. Neutralizing tumor-promoting chronic inflammation: a magic bullet? Science. 2013;339(6117):286-291.

37. Mantovani A, Allavena P, Sica A, Balkwill F. Cancer-related inflammation. Nature. 2008;454(7203):436-444.

38. Hanahan D, Weinberg RA. Hallmarks of cancer: the next generation. Cell. 2011;144(5):646-674.

39. Bonavita E, et al. PTX3 is an extrinsic oncosuppressor regulating complement-dependent inflammation in cancer. Cell. 2015;160(4):700-714.

40. Pio R, Ajona D, Lambris JD. Complement inhibition in cancer therapy. Semin Immunol. 2013;25(1):54-64.

41. Nitta H, Murakami Y, Wada Y, Eto M, Baba H, Imamura $\mathrm{T}$. Cancer cells release anaphylatoxin C5a from $\mathrm{C} 5$ by serine protease to enhance invasiveness. Oncol Rep. 2014;32(4):1715-1719.

42. Bulla R, et al. C1q acts in the tumour microenvironment as a cancer-promoting factor independently of complement activation. Nat Commun. 2016;7:10346.

43. Fishelson Z, Donin N, Zell S, Schultz S, Kirschfink M. Obstacles to cancer immunotherapy: expression of membrane complement regulatory proteins (mCRPs) in tumors. Mol Immunol. 2003;40(2-4):109-123.

44. Gancz D, Fishelson Z. Cancer resistance to complement-dependent cytotoxicity (CDC): Problem-oriented research and development. Mol Immunol. 2009;46(14):2794-2800.

45. Grivennikov SI, Greten FR, Karin M. Immunity, inflammation, and cancer. Cell. 2010;140(6):883-899.
46. Ricklin D, Hajishengallis G, Yang K, Lambris JD. Complement: a key system for immune surveillance and homeostasis. Nat Immunol. 2010;11(9):785-797.

47. Gorter A, Meri S. Immune evasion of tumor cells using membrane-bound complement regulatory proteins. Immunol Today. 1999;20(12):576-582.

48. Nishioka K, Kawamura K, Hirayama T, Kawashima T, Shimada K. The complement system in tumor immunity: significance of elevated levels of complement in tumor bearing hosts. Ann N Y Acad Sci. 1976;276:303-315.

49. Chow MT, Moller A, Smyth MJ. Inflammation and immune surveillance in cancer. Semin Cancer Biol. 2012;22(1):23-32.

50. Markiewski MM, Lambris JD. Is complement good or bad for cancer patients? A new perspective on an old dilemma. Trends Immunol. 2009;30(6):286-292.

51. Taylor RP, Lindorfer MA. Cytotoxic mechanisms of immunotherapy: Harnessing complement in the action of anti-tumor monoclonal antibodies. Semin Immunol. 2016;28(3):309-316.

52. Di Gaetano N, et al. Complement activation determines the therapeutic activity of rituximab in vivo. J Immunol. 2003;171(3):1581-1587.

53. Wang SY, Racila E, Taylor RP, Weiner GJ. NK-cell activation and antibody-dependent cellular cytotoxicity induced by rituximab-coated target cells is inhibited by the $\mathrm{C} 3 \mathrm{~b}$ component of complement. Blood. 2008;111(3):1456-1463.

54. Wang SY, et al. Depletion of the C3 component of complement enhances the ability of rituximab-coated target cells to activate human NK cells and improves the efficacy of monoclonal antibody therapy in an in vivo model. Blood. 2009;114(26):5322-5330.

55. Pedersen IM, Buhl AM, Klausen P, Geisler CH, Jurlander J. The chimeric anti-CD20 antibody rituximab induces apoptosis in B-cell chronic lymphocytic leukemia cells through a p38 mitogen activated protein-kinase-dependent mechanism. Blood. 2002;99(4):1314-1319.

56. Kheirallah S, et al. Rituximab inhibits B-cell receptor signaling. Blood. 2010;115(5):985-994.

57. Prang N, et al. Cellular and complement-dependent cytotoxicity of Ep-CAM-specific monoclonal antibody MT201 against breast cancer cell lines. Br JCancer. 2005;92(2):342-349.

58. Ward PA. Functions of C5a receptors. J Mol Med (Berl). 2009;87(4):375-378.

59. Perianayagam MC, Balakrishnan VS, King AJ, Pereira BJ, Jaber BL. C5a delays apoptosis of human neutrophils by a phosphatidylinositol 3-kinase-signaling pathway. Kidney Int . 2002;61(2):456-463.

60. Kurihara R, et al. C5a promotes migration, proliferation, and vessel formation in endothelial cells. Inflamm Res. 2010;59(8):659-666.

61. Cao Q, McIsaac SM, Stadnyk AW. Human colonic epithelial cells detect and respond to C5a via apically expressed C5aR through the ERK pathway. Am J Physiol Cell Physiol. 2012;302(12):C1731-C1740.

62. Zhou X, et al. Complement 3 activates the renal renin-angiotensin system by induction of epithelial-to-mesenchymal transition of the nephrotubulus in mice. Am J Physiol Renal Physiol. 2013;305(7):F957-F967. 
63. Cho MS, et al. Complement component 3 is regulated by TWIST1 and mediates epithelial-mesenchymal transition. JImmunol. 2016;196(3):1412-1418.

64. Niculescu F, Badea T, Rus H. Sublytic C5b-9 induces proliferation of human aortic smooth muscle cells: role of mitogen activated protein kinase and phosphatidylinositol 3-kinase. Atherosclerosis. 1999;142(1):47-56.

65. Soane L, Cho HJ, Niculescu F, Rus H, Shin ML. C5b-9 terminal complement complex protects oligodendrocytes from death by regulating Bad through phosphatidylinositol 3-kinase/Akt pathway. J Immunol. 2001;167(4):2305-2311.

66. Kraus S, Seger R, Fishelson Z. Involvement of the ERK mitogen-activated protein kinase in cell resistance to complement-mediated lysis. Clin Exp Immunol. 2001;123(3):366-374.

67. Markiewski MM, et al. Modulation of the antitumor immune response by complement. Nat Immunol. 2008;9(11):1225-1235.

68. Peranzoni E, et al. Myeloid-derived suppressor cell heterogeneity and subset definition. Curr Opin Immunol. 2010;22(2):238-244.

69. Ochando JC, Chen SH. Myeloid-derived suppressor cells in transplantation and cancer. Immunol Res. 2012;54(1-3):275-285.

70. Kusmartsev S, Nefedova Y, Yoder D, Gabrilovich DI. Antigen-specific inhibition of $\mathrm{CD}^{+} \mathrm{T}$ cell response by immature myeloid cells in cancer is mediated by reactive oxygen species. J Immunol. 2004;172(2):989-999.

71. Nunez-Cruz S, et al. Genetic and pharmacologic inhibition of complement impairs endothelial cell function and ablates ovarian cancer neovascularization. Neoplasia. 2012;14(11):994-1004.

72. Connolly DC, et al. Female mice chimeric for expression of the simian virus $40 \mathrm{TAg}$ under control of the MISIIR promoter develop epithelial ovarian cancer. Cancer Res. 2003;63(6):1389-1397.

73. Cho MS, et al. Autocrine effects of tumor-derived complement. Cell Rep. 2014;6(6):1085-1095.

74. Wang Y, et al. et al. Autocrine complement inhibits IL10-dependent T-cell-mediated antitumor immunity to promote tumor progression. Cancer Discov. 2016;6(9):1022-1035.

75. Mamidi S, Höne S, Kirschfink M. The complement system in cancer: Ambivalence between tumour destruction and promotion. Immunobiology. 2017;222(1):45-54.

76. Shi XX, Zhang B, Zang JL, Wang GY, Gao MH. CD59 silencing via retrovirus-mediated RNA interference enhanced complement-mediated cell damage in ovary cancer. Cell Mol Immunol. 2009;6(1):61-66.

77. Donin N, Jurianz K, Ziporen L, Schultz S, Kirschfink M, Fishelson Z. Complement resistance of human carcinoma cells depends on membrane regulatory proteins, protein kinases and sialic acid. Clin Exp Immunol. 2003;131(2):254-263.
78. Lovisa S, et al. Epithelial-to-mesenchymal transition induces cell cycle arrest and parenchymal damage in renal fibrosis. Nat Med. 2015;21(9):998-1009.

79. Kalluri R, Weinberg RA. The basics of epithelial-mesenchymal transition. JClin Invest. 2009;119(6):1420-1428.

80. Wan J, Zhou X, Cui J, Zou Z, Xu Y, You D. Role of complement 3 in TNF- $\alpha$-induced mesenchymal transition of renal tubular epithelial cells in vitro. Mol Biotechnol. 2013;54(1):92-100.

81. Zhou M, Ma H, Lin H, Qin J. Induction of epithelial-to-mesenchymal transition in proximal tubular epithelial cells on microfluidic devices. Biomaterials. 2014;35(5):1390-1401.

82. Goswami MT, et al. Regulation of complement-dependent cytotoxicity by TGF- $\beta$-induced epithelial-mesenchymal transition. Oncogene. 2016;35(15):1888-1898.

83. Macor P, Tedesco F. Complement as effector system in cancer immunotherapy. Immunol Lett. 2007;111(1):6-13.

84. Zhao WP, Zhu B, Duan YZ, Chen ZT. Neutralization of complement regulatory proteins CD55 and CD59 augments therapeutic effect of herceptin against lung carcinoma cells. Oncol Rep. 2009;21(6):1405-1411.

85. Hu W, et al. Human CD59 inhibitor sensitizes rituximab-resistant lymphoma cells to complement-mediated cytolysis. Cancer Res. 2011;71(6):2298-2307.

86. Laverdière I, et al. Complement cascade gene expression defines novel prognostic subgroups of acute myeloid leukemia. Exp Hematol. 2016;44(11):1039-1043.e10.

87. Abdelbaset-Ismail A, et al. Activation of the complement cascade enhances motility of leukemic cells by downregulating expression of HO-1 [published online ahead of print August 26, 2016]. Leukemia. https://doi.org/10.1038/leu.2016.198.

88. Zuber J, Fakhouri F, Roumenina LT, Loirat C, Frémeaux-Bacchi V, French Study Group for aHUS/C3G. Use of eculizumab for atypical haemolytic uraemic syndrome and C3 glomerulopathies. Nat Rev Nephrol. 2012;8(11):643-657.

89. Cugno M, et al. Complement functional tests for monitoring eculizumab treatment in patients with atypical hemolytic uremic syndrome. J Thromb Haemost. 2014;12(9):1440-1448.

90. Canales NA, et al. A1BG and C3 are overexpressed in patients with cervical intraepithelial neoplasia III. Oncol Lett. 2014;8(2):939-947.

91. Lin K, et al. Complement component 3 is a prognostic factor of non-small cell lung cancer. Mol Med Rep. 2014;10(2):811-817.

92. Bouwens TA, Trouw LA, Veerhuis R, Dirven CM, Lamfers ML, Al-Khawaja H. Complement activation in Glioblastoma multiforme pathophysiology: evidence from serum levels and presence of complement activation products in tumor tissue. J Neuroimmunol. 2015;278:271-276.
93. Nabizadeh JA, et al. The complement C3a receptor contributes to melanoma tumorigenesis by inhibiting neutrophil and $\mathrm{CD} 4^{+} \mathrm{T}$ cell responses. J Immunol. 2016;196(11):4783-4792.

94. Habermann JK, et al. Increased serum levels of complement C3a anaphylatoxin indicate the presence of colorectal tumors. Gastroenterology. 2006;131(4):1020-1029; quiz 1284.

95. Bjørge L, et al. Ascitic complement system in ovarian cancer. Br JCancer. 2005;92(5):895-905.

96. Corrales L, et al. Anaphylatoxin C5a creates a favorable microenvironment for lung cancer progression. J Immunol. 2012;189(9):4674-4683.

97. Nitta H, et al. Enhancement of human cancer cell motility and invasiveness by anaphylatoxin $\mathrm{C} 5 \mathrm{a}$ via aberrantly expressed C5a receptor (CD88). Clin Cancer Res. 2013;19(8):2004-2013.

98. Baatrup G, Qvist N, Junker A, Larsen KE, Zimmermann-Nielsen C. Activity and activation of the complement system in patients being operated on for cancer of the colon. Eur J Surg. 1994;160(9):503-510.

99. Ajona D, et al. Complement activation product C4d in oral and oropharyngeal squamous cell carcinoma. Oral Dis. 2015;21(7):899-904.

100.Chen J, Yang WJ, Sun HJ, Yang X, Wu YZ. C5b-9 staining correlates with clinical and tumor stage in gastric adenocarcinoma. Appl Immunohistochem MolMorphol.2016;24(7):470-475.

101. Niculescu F, Rus HG, Retegan M, Vlaicu R. Persistent complement activation on tumor cells in breast cancer. Am J Pathol. 1992;140(5):1039-1043.

102. Yamakawa M, et al. Protection of thyroid cancer cells by complement-regulatory factors. Cancer. 1994;73(11):2808-2817.

103. Lucas SD, Ek B, Rask L, Rastad J, Akerström G, Juhlin C. Identification of a $35 \mathrm{kD}$ tumor-associated autoantigen in papillary thyroid carcinoma. Anticancer Res. 1996;16(5A):2493-2496.

104.Bulla R, et al. C1q acts in the tumour microenvironment as a cancer-promoting factor independently of complement activation. Nat Commun. 2016;7:10346.

105. Ytting H, Jensenius JC, Christensen IJ, Thiel $\mathrm{S}$, Nielsen HJ. Increased activity of the mannan-binding lectin complement activation pathway in patients with colorectal cancer. Scand J Gastroenterol. 2004;39(7):674-679.

106.Ytting H, Christensen IJ, Thiel S, Jensenius JC, Nielsen HJ. Serum mannan-binding lectinassociated serine protease 2 levels in colorectal cancer: relation to recurrence and mortality. Clin Cancer Res. 2005;11(4):1441-1446.

107. Riihilä PM, et al. Complement factor H: a biomarker for progression of cutaneous squamous cell carcinoma. J Invest Dermatol. 2014;134(2):498-506.

108. Cheng ZZ, et al. Complement factor $\mathrm{H}$ as a marker for detection of bladder cancer. Clin Chem. 2005;51(5):856-863. 